\title{
Les services de photocopie dans les bibliothèques : la Cour suprême du Canada se prononce
}

\author{
Un commentaire de l'arrêt CCH Canadienne ltée c. Barreau du \\ Haut-Canada
}

\section{Photocopy Services in Libraries: The Supreme Court of Canada Renders a Decision}

\author{
A Commentary of the Decision in the Case of CCH Canada Ltd, c. Law \\ Society of Upper Canada
}

\section{Los servicios de fotocopia en las bibliotecas: la Corte Suprema de Canadá se pronuncia al respecto. \\ Comentario del fallo de CCH Canadienne Ltée. c. Colegio de Abogados de Ontario}

Jules Larivière

Volume 51, numéro 3, juillet-septembre 2005

URI : https://id.erudit.org/iderudit/1029495ar

DOI : https://doi.org/10.7202/1029495ar

Aller au sommaire du numéro

Éditeur(s)

Association pour l'avancement des sciences et des techniques de la documentation (ASTED)

ISSN

0315-2340 (imprimé)

2291-8949 (numérique)

Découvrir la revue

Citer cet article

Larivière, J. (2005). Les services de photocopie dans les bibliothèques : la Cour suprême du Canada se prononce : un commentaire de l'arrêt $C C H$ Canadienne ltée c. Barreau du Haut-Canada. Documentation et bibliothèques, 51(3), 165-174. https://doi.org/10.7202/1029495ar
Résumé de l'article

En mars 2004, la Cour suprême du Canada a rendu une importante décision concernant les services de photocopie dans les bibliothèques, en statuant que ce service offert par une bibliothèque constituait une utilisation équitable. L'auteur présente l'historique de cette cause ainsi qu'une analyse détaillée qui permet d'en dégager les conséquences. Il rappelle enfin l'importance pour toutes les bibliothèques d'établir des politiques d'accès et des lignes directrices claires afin d'encadrer les services de livraison de documents.
Tous droits réservés (c) Association pour l'avancement des sciences et des techniques de la documentation (ASTED), 2005
Ce document est protégé par la loi sur le droit d'auteur. L'utilisation des services d'Érudit (y compris la reproduction) est assujettie à sa politique d'utilisation que vous pouvez consulter en ligne.

https://apropos.erudit.org/fr/usagers/politique-dutilisation/ 


\title{
Les services de photocopie dans les bibliothèques : la Cour suprême du Canada se prononce
}

\author{
Un commentaire de l'arrêt CCH Canadienne ltée c. Barreau du \\ Haut-Canada
}

JULES LARIVIÈRE*

\begin{abstract}
RÉSUMÉ | ABSTRACTS | RESUMEN
En mars 2004, la Cour suprême du Canada a rendu une importante décision concernant les services de photocopie dans les bibliothèques, en statuant que ce service offert par une bibliothèque constituait une utilisation équitable. L'auteur présente l'historique de cette cause ainsi qu'une analyse détaillée qui permet d'en dégager les conséquences. Il rappelle enfin l'importance pour toutes les bibliothèques d'établir des politiques d'accès et des lignes directrices claires afin d'encadrer les services de livraison de documents.
\end{abstract}

\section{Photocopy Services in Libraries: The Supreme Court of Canada Renders a Decision A Commentary of the Decision in the Case of CCH Canada Ltd. c. Law Society of Upper Canada}

In March 2004, the Supreme Court of Canada rendered an important decision regarding photocopy services in libraries: it stated that the service constituted fair use. The author outlines the development of this case and provides a detailed analysis of its impact. He reminds libraries that they must develop clear access policies and guidelines that better manage document delivery.

\section{Los servicios de fotocopia en las bibliotecas : la Corte Suprema de Canadá se pronuncia al respecto. Comentario del fallo de CCH Canadienne Ltée. $c$. Colegio de Abogados de Ontario}

En marzo de 2004, la Corte Suprema de Canadá tomó una decisión importante con respecto a los servicios de fotocopia en las bibliotecas, estableciendo que el servicio ofrecido en estas instituciones es de uso equitativo. El autor expone el origen de esta cuestión y un análisis detallado que permite destacar sus consecuencias. Asimismo, recuerda a todas las bibliotecas la importancia de establecer políticas de acceso y directrices claras a fin de especificar los servicios de entrega de los documentos.

\section{INTRODUCTION}

E 4 MARS 2004, la Cour suprême du Canada a rendu une décision ${ }^{1}$ concernant le service de photocopie qu'offre la Bibliothèque du barreau du Haut-Canada aux membres de la profession juridique de l'Ontario. Il s'agit d'une décision importante pour l'ensemble du monde des bibliothèques canadiennes, puisque la Cour, dans une décision unanime, a statué que le service de livraison de photocopies offert par cette bibliothèque constituait une utilisation équitable et ne violait pas le droit d'auteur des éditeurs juridiques canadiens qui avaient déposé la plainte. De plus, la Cour a établi qu'une bibliothèque ne peut être tenue responsable des reproductions illicites effectuées à l'aide des photocopieurs libre-service qu'elle met à la disposition de ses usagers. Il faut bien noter ici que l'obligation légale d'afficher des avertissements appropriés demeure, tel que prévu dans la loi canadienne sur le droit d'auteur. Le plus haut tribunal du pays a également décidé que la transmission par télécopieur d'une œuvre protégée à un seul destinataire ne constitue pas une «une transmission au public» au sens de la Loi sur le droit d'auteur et n'est donc pas une activité protégée par la loi.

Les décisions judiciaires touchant directement les bibliothèques sont plutôt rares. Lorsqu'une d'elles émane de la Cour suprême du Canada, c'est un événement qui mérite qu'on s'y arrête. Considérant en effet qu'il s'agit d'une décision émanant de l'instance judiciaire de dernier recours au Canada, l'application de la Loi sur le droit d'auteur dans les bibliothèques s'en trouve ainsi précisée. C'est aussi une décision qui a des conséquences directes sur la prestation des services documentaires dans l'ensemble des bibliothèques canadiennes. Il y a donc lieu d'en faire une analyse détaillée pour bien en comprendre tout le sens. Même si les faits de la cause se situent à l'intérieur d'un environnement juridique, tant au niveau de la documentation que de la bibliothèque, la Cour suprême a quand même établi certains principes qui sont valables pour toute catégorie de documentation et de bibliothèque. 1. CCH Canadienne Itée c. Barreau du Haut-Canada, [2004], 1. Recueils des arrêts de
la Cour suprême du Canada, p. 339 . 


\section{LES FAITS EN CAUSE}

La Bibliothèque du barreau de l'Ontario est une des bibliothèques de droit les plus importantes au Canada. En plus de mettre à la disposition de sa clientèle des appareils à photocopier, elle offre un service de photocopie par son personnel et de transmission par télécopieur de documentation juridique aux membres de la profession juridique ontarienne, y compris les juges et les étudiants en droit. Parmi la documentation photocopiée, on retrouve des textes de lois et de règlements, des décisions des tribunaux telles que publiées dans des rapports judiciaires, des articles de revues juridiques et des extraits de monographies ou d'ouvrages de référence, toujours dans le domaine du droit. La bibliothèque facture des frais pour chacune des transactions de façon à couvrir ses coûts de service. La bibliothèque est considérée comme faisant partie d'un organisme à but non lucratif au sens de la Loi sur le droit d'auteur ${ }^{2}$. La prestation de ce service documentaire est encadrée par une Politique d'accès au droit et par des Lignes directrices. La politique et les lignes directrices stipulent qu'on ne peut photocopier qu'un seul exemplaire de documents juridiques, et ce, uniquement dans un but de recherche, d'analyse, d'étude privée, de critique ou d'utilisation devant un tribunal ou pour une procédure gouvernementale. Elles prévoient également que le Barreau ne fera pas de photocopies pour des motifs autres que ceux désignés dans le formulaire de demande. On y indique aussi que toute demande équivalant à plus de $5 \%$ du nombre de pages d'un livre ou de plus de deux extraits d'un document sera évaluée par un bibliothécaire de référence et pourrait être refusée s'il est jugé qu'il s'agit d'un extrait trop substantiel ${ }^{3}$.

Pendant plusieurs années, les maisons d'édition juridique Canada - Commerce Clearing House ( $\mathrm{CCH}$ Canadienne ltée c.), Carswell et Canada Law Book - ont fait parvenir des avis au barreau ontarien l'enjoignant de cesser ces pratiques qu'elles considéraient comme une violation de leurs droits d'auteur. Devant le refus du barreau du Haut-Canada de répondre favorablement à leur demande, les trois éditeurs juridiques ont entamé des procédures devant la Cour fédérale.

Les éditeurs ont préparé leur cause en demandant à un de leurs services juridiques de faire une demande à la Bibliothèque du barreau du Haut-Canada et de se faire tranmettre par télécopieur les photocopies de trois décisions judiciaires telles que publiées dans le Dominion Law Reports, des extraits d'un volume qui commentait le texte d'une loi et un article tiré d'un manuel de droit. Il faut noter que ces demandes ont été faites avant qu'entrent en vigueur les exceptions pour les bibliothèques qui sont maintenant prévues dans la loi canadienne sur le droit d'auteur.

\section{LES QUESTIONS EN LITIGE}

Compte tenu des prétentions des parties, le tribunal a été appelé à étudier de nombreuses questions de droit. Mais le présent article s'en tient uniquement à celles qui touchent directement les bibliothèques. Même si les faits étaient tous reliés spécifiquement à une bibliothèque de droit, la décision rendue a des répercussions importantes pour l'ensemble des bibliothèques. Ainsi, par exemple, le juge devait se prononcer sur le degré de responsabilité d'une bibliothèque quant aux violations du droit d'auteur qui peuvent survenir, découlant de l'utilisation des machines à photocopier qu'on retrouve dans les bibliothèques. De plus, étant donné la défense du Barreau, à savoir que leur service de photocopie assuré par le personnel de la bibliothèque constituait une "utilisation équitable» des œuvres reproduites, le tribunal devait donc préciser le sens de cette notion juridique propre à la Loi sur le droit d'auteur et plus spécifiquement dans le contexte des bibliothèques. En outre, étant donné le genre de documentation en cause, à savoir des jugements des différents tribunaux canadiens, des résumés jurisprudentiels, qui sont préparés à partir des faits d'une cause et de l'identification des points de droit qui y sont soulevés, et des index analytiques qui sont des ouvrages de référence permettant de relier des points de droit à des décisions spécifiques, la question de l'originalité d'une œuvre était une autre question importante à étudier. Les deux parties s'entendaient au départ pour admettre que les jugements rédigés par un juge étaient protégés par un droit d'auteur, celuici appartenant à la Couronne, c'est-à-dire au gouvernement, compte tenu de la structure constitutionnelle $\mathrm{du}$ pays. Mais les éditeurs juridiques prétendaient qu'en ajoutant un résumé jurisprudentiel, un sommaire de jurisprudence et des informations supplémentaires à un jugement original tel que rédigé par un juge, ils créaient une nouvelle œuvre. Il fallait donc déterminer si cette nouvelle œuvre était suffisamment originale pour être à son tour protégée par un droit d'auteur distinct.

Ils alléguaient également qu'en emmagasinant des textes dans la mémoire d'un télécopieur, on effectuait des copies d'une œuvre protégée, et qu'en transmettant les copies par télécopieur, la bibliothèque effectuait une diffusion publique d'une œuvre littéraire, ce que seul le détenteur des droits peut faire en vertu de la Loi sur le droit d'auteur.

2. Loi sur le droit d'auteur, L.R.C. 1985 , c. C-42, art. 2

3. Access to the Law Policy and Guidelines, en ligne: The Law Society of Upper Canada. Great Library. http://library.lsuc.on.ca/GL/home.htm (date d'accès: 31 décembre 2004). 


\section{LES NOTIONS D'ORIGINALITÉ, D'UTILISATION ÉQUITABLE ET D'AUTORISATION EN DROIT D'AUTEUR CANADIEN}

Afin de bien comprendre la portée de la décision, il y a lieu d'expliquer quelque peu la notion d'originalité d'une œuvre, celle d'utilisation équitable ainsi que la notion d'autorisation, trois questions sur lesquelles la Cour a dû se prononcer.

L'article 5 (1) de la loi canadienne sur le droit d'auteur stipule que "le droit d'auteur existe au Canada, pendant la durée mentionnée ci-après, sur toute ouvre littéraire, dramatique, musicale ou artistique originale». L'originalité d'une œuvre est donc une condition essentielle à sa protection. En droit d'auteur, pour être originale, une œuvre ne doit pas être une copie d'une autre œuvre, c'est-à-dire qu'elle doit se distinguer substantiellement de toute autre œuvre. Mais il faut aussi préciser que ce qui est original ne doit pas nécessairement être novateur ou unique ${ }^{4}$. Mais l'œuvre doit aussi être le résultat d'un travail qui a nécessité l'exercice d'un certain talent et d'un certain jugement ainsi que d'un certain effort intellectuel de la part de son créateur. Il faut comprendre ici que ce dernier aspect faisait l'objet d'une certaine controverse parmi les juristes canadiens, ce qui rend la présente décision d'autant plus importante. En effet depuis la décision Télé-Direct (Publications) Inc. c. American Business Information Inc. ${ }^{5}$ de la Cour fédérale d'appel, certains experts prétendaient qu'en plus d'un certain degré de travail, d'effort, de jugement ou de talent, l'arrêt Télé-Direct avait ajouté un nouvel élément à l'originalité, à savoir une nécessité d'un certain degré de créativité.

Quant à la notion d'utilisation équitable, on la retrouve spécifiquement mentionnée à l'article 29 de la Loi sur le droit d'auteur qui se lit comme suit: «L'utilisation équitable d'une ouvre ou de tout autre objet du droit d'auteur aux fins d'étude privée ou de recherche ne constitue pas une violation du droit d'auteur." Le principe de base sur lequel repose toute législation sur le droit d'auteur est la nécessité de maintenir un équilibre entre les droits reconnus des créateurs, à savoir des droits économiques et des droits moraux, et les besoins légitimes, mais non abusifs, des utilisateurs des œuvres. D'une part, le droit d'auteur fournit au créateur une forme de monopole sur son œuvre. En effet, l'article 3(1) de la loi précise que le droit de l'auteur sur son œuvre comporte son droit exclusif d'en produire ou reproduire la totalité ou une partie importante. L'article vise par la suite toute une série d'activités que seul le créateur peut réaliser ou autoriser à

4. N. Tamaro, Loi sur le droit d'auteur: texte annoté, $6^{\mathrm{e}}$ éd., Scarborough, Ont, Carswell, 2003, p. 171.

5. [1998], 2 Recueil des arrêts de la Cour fédérale du Canada, p. 22 accomplir, ce qui signifie que «l'auteur a le droit de s'opposer et d'interdire à quiconque d'exercer un droit protégé par le paragraphe $3(1)^{6} »$. D'autre part, même si le premier objectif d'une législation sur le droit d'auteur est de protéger les droits du créateur sur son œuvre, elle ne doit cependant pas empêcher une utilisation de celle-ci ne lui causant pas un préjudice. Comme l'écrit Catherine Bergeron, dans son article pré-cité:

«[...] si la loi ne visait que cet objectif [i.e. protéger le créateur] et se voulait la gardienne des droits exclusifs de l'auteur, n'y aurait-il pas là un débalancement démesuré entre l'auteur et le reste de la "population créatrice» en faveur de ce premier? Il faut répondre par l'affirmative à cette question. En effet, si une telle exception [i.e. l'utilisation équitable] n'existait pas dans la loi, cela aurait pour effet de freiner outrageusement l'élan créateur d'autres auteurs. Soit qu'ils ne pourraient tout simplement pas utiliser une partie d'une ouvre protégée, soit qu'ils se verraient obligés de s'engager dans un long processus d'autorisation de l'auteur pour l'utilisation d'une partie de son ouvre ${ }^{7} . »$

C'est dans ce contexte que les législations nationales prévoient pour la plupart, à des degrés divers, que certaines exceptions puissent venir limiter la protection accordée par le droit d'auteur et permettre d'avoir un accès facilité aux œuvres protégées. C'est ainsi que le droit français parlera de la " copie privée ${ }^{8}$ ", le droit américain du «fair use ${ }^{9}$ » (usage équitable), et le droit australien, britannique et canadien, de l'«utilisation équitable» (fair dealing). Mais dans tous les cas il s'agit d'exceptions qui sont bien encadrées, les lois précisant les circonstances dans lesquelles on peut s'en prévaloir.

Quant à la notion d'autorisation, elle est précisée à la fin de l'article 3 (1) qui stipule que le détenteur d'un droit d'auteur a le droit exclusif d'autoriser une personne à utiliser son œuvre pour accomplir les actes que lui seul peut accomplir. On peut donc violer le droit d'auteur d'un créateur en permettant à une personne d'accomplir un acte alors qu'on ne possède pas l'autorité pour le faire. C'est ainsi que les éditeurs juridiques prétendaient que le barreau de l'Ontario, en mettant des machines à photocopier à la disposition des usagers de leur bibliothèque, autorisait ceux-ci à reproduire des œuvres protégées sans avoir le droit de le faire.

6. C. Bergeron, «Fair dealing canadien et fair use américain: une analyse de l'exception d'utilisation équitable en matière de droit d'auteur", Cahiers de propriété intellectuelle, vol. $13, \mathrm{n}^{\circ} 2,2001, \mathrm{p} .275$

7. Ibid., p. 279.

8. Art. L. 122-5, Code de propriété intellectuelle.

9. Copyright Act, 17 U.S.C. \$107. 


\section{L'HISTORIQUE DE LA DÉCISION}

\section{À la Cour fédérale, Division de première instance}

La Cour fédérale, Division de première instance, a entendu la cause en novembre 1998. Un an plus tard, soit le 9 novembre 1999, le juge Gibson rendait un jugement ${ }^{10}$ controversé à un point tel que les deux parties ont décidé de faire appel. Dans un premier temps, le jugement considère que les décisions judiciaires telles qu'enrichies par les éditeurs juridiques ne constituent pas des œuvres «nouvelles» suffisamment "originales" pour se voir octroyer un nouveau droit d'auteur. Pour le juge Gibson, bien qu'il admette qu'une somme importante de travail, d'habileté et de jugement soit nécessaire pour préparer des résumés jurisprudentiels, il y manque quand même un élément de création qu'il juge nécessaire pour qu'une œuvre soit originale. Au paragraphe 139 de la décision, le juge écrit:

«[...] la préparation des décisions judiciaires publiées, incluant les sommaires, les mots clés, les références parallèles, les titres courants et d'autres points ajoutés par l'éditeur, comporte énormément d'efforts, d'habileté et de jugement, mais je suis convaincu que l'ensemble du processus, et plus particulièrement les éléments qui demandent de l'habileté et du jugement, sont dépourvus de "l'imagination" ou de "létincelle créatrice" que je juge jusquà maintenant essentielles à une conclusion d'originalité»

Dans un deuxième temps, le juge Gibson est d'avis que la défense d'utilisation équitable ne peut être invoquée par le barreau du Haut-Canada. Selon lui, au moment où les photocopies ont été faites, soit en janvier et février 1992 et 1993 ainsi qu'en janvier 1998, une bibliothèque ne pouvait elle-même prétendre à une défense d'utilisation équitable, celle-ci étant exclusivement réservée à une personne effectuant elle-même de la recherche. Il appuie son argumentation sur le fait que le législateur a justement modifié la Loi sur le droit d'auteur en 1999 pour prévoir une exception spécifique pour une bibliothèque qui accomplit un acte comme photocopier, par exemple, pour une personne qui peut elle-même l'accomplir dans le cadre d'une utilisation équitable ${ }^{11}$.

Quant à la question de la transmission des copies par télécopieur, le juge considère qu'il s'agit bien d'une communication d'une œuvre littéraire par télécommunication, mais qu'en transmettant l'œuvre à un seul

10. CCH Canadienne ltée c. Barreau du Haut-Canada ( ${ }^{\mathrm{re}}$ inst.), [200o] 2 Recueil des arrêts de la Cour fédérale du Canada, p. 451.

11. Supra note 2, à l'art. 30.2 (1) destinataire, il ne s'agissait pas d'une communication "au public" au sens de la Loi sur le droit d'auteur.

Enfin, sur la question de la responsabilité de la bibliothèque face aux violations possibles du droit d'auteur découlant de l'utilisation des photocopieuses libre-service, le juge Gibson refuse de se prononcer compte tenu, selon lui, que "la preuve soumise quant à l'utilisation réelle des photocopieuses individuelles de la grande bibliothèque est remarquablement limitée ${ }^{12} »$.

\section{À la Cour fédérale d'appel}

Les deux parties en ayant donc appelé, ce fut à la Cour fédérale d'appel de se prononcer sur les questions soulevées par les deux parties. Dans une décision fort bien documentée de 132 pages, rendue le 14 mai $\mathbf{2 0 O 2}^{13}$, les juges Linden, Sharlow et Rothstein renversent la décision du juge de première instance sur la question de l'originalité en affirmant que les décisions judiciaires telles que publiées par les éditeurs juridiques sont des œuvres originales au sens de la Loi sur le droit d'auteur et méritent ainsi d'être protégées. Selon eux, «la condition préalable traditionnelle pour qu'il existe un droit d'auteur en droit anglo-canadien est qu'une ouvre soit produite de façon indépendante et non copiée de quelqu'un d'autre ${ }^{14}{ }^{\prime}$. Ils estiment que le juge Gibson a mal interprété l'arrêt Télé-Direct en considérant que cet arrêt ajoutait de nouvelles exigences «d'imagination» et "d'étincelles créatrices» pour qu'une œuvre soit considérée comme originale.

Un aspect intéressant de la décision de la Cour fédérale d'appel est celui concernant la protection à accorder à des parties d'une œuvre. Le Barreau ayant allégué "que les sommaires qui accompagnent les décisions judiciaires publiées ne sont pas des cuvres, mais seulement des parties d'ouvres ${ }^{15}$ ", le tribunal a dû examiner la problématique de ce qui constitue une partie d'œuvre suffisamment originale pour mériter d'être protégée. Il s'agit là d'une question importante, puisqu'elle permet de savoir quelles parties d'œuvres on peut reproduire sans violer le droit d'auteur, et peut aider à déterminer ce qu'est "une partie importante» dont parle l'article 3(1) de la Loi sur le droit d'auteur. Pour le tribunal:

"[i]l faut établir si l'ouvre [ou la partie d'œuvre] en question peut exister en dehors $d u$ contexte dans lequel elle est publiée, communiquée, exposée, exécutée ou autrement diffusée. La production qui est distinctive et peut raisonnablement exister en tant que telle peut être considérée comme une ouvre en soi plutôt

12. Supra note $10, \$ 191$,

13. CCH Canadienne ltée c. Barreau du Haut-Canada (C.A.), [2002] 4 Recueil des arrêts de la Cour fédérale d'appel du Canada, p. 213.

14. Ibid., $\$ 36$

15. Ibid., $\$ 61$. 
qu'une partie d'une autre ouvre. Mais la production qui dépend tellement des éléments qui l'entourent qu'elle perd tout son sens sans eux, ou dont l'utilité disparaît en grande partie lorsqu'elle est séparée du contexte dans lequel elle est diffusée ne sera en revanche qu'une simple partie d'ouvre.»16.

C'est ainsi que la Cour fédérale d'appel conclut que les sommaires de jurisprudence sont des «parties d'œuvres» originales et donc protégées. Poursuivant son examen de la question, la Cour établit que les index sont protégés puisqu'il faut quand même un certain niveau d'habileté et de jugement dans le choix et l'organisation des données.

Sur la question de l'utilisation équitable, les juges de la Cour fédérale d'appel refusent d'abord de se prononcer sur les reproductions des onze œuvres spécifiques qui ont été utilisées par les éditeurs pour déposer leur plainte. Pour les juges,

" [...] il n'y a guère eu d'éléments de preuve ou de débats écrits ou oraux concernant léquité d'utilisations particulières. À l'instar des allégations de violation, les arguments visaient plutôt généralement l'incidence des activités du Barreau en vertu de sa Politique d'accès à l'information juridique, et non pas des actes ou des événements précis. Puisqu'une appréciation véritable de l'utilisation équitable ne peut se faire que dans un contexte factuel précis, je ne peux pas me prononcer définitivement sur l'équité de l'utilisation qu'a faite le Barreau en l'espèce des onze ouvres spécifiques des éditeurs».17

Cela étant dit, les trois juges considèrent que la question de l'utilisation équitable est une question centrale dans ce litige, puisqu'elle était au cœur des moyens de défense du Barreau. Pour cette raison, ils considèrent qu'ils doivent se prononcer sur le sens du concept d'utilisation équitable que la loi ne définit pas. Dans un premier temps, le tribunal analyse les deux raisons pour justifier une utilisation équitable tel que mentionnée à l'article 29, à savoir que "[l]'utilisation équitable d'une oeuvre aux fins d'étude privée ou de recherche ne constitue pas une violation du droit d'auteur». Les juges font remarquer que le législateur a qualifié le mot étude par le terme privée, tandis que le mot recherche ne l'est pas. Pour eux, "la décision $d u$ législateur de qualifier expressément l'étude" et non pas la "recherche" indique une intention de permettre toute recherche équitable, que ce soit dans un cadre privé ou non ${ }^{18}$ ". Il s'agit là d'une décision très importante parce qu'elle ouvre la porte à la prétention à l'utilisation équitable pour la recherche dans un contexte commercial ou dans un but lucratif, ce que les éditeurs prétendaient être une fin non admissible dans le contexte de l'article 29. Dans un deuxième temps, le tribunal s'est demandé si l'utilisation équitable ne s'appliquait que lorsqu'on effectue une recherche ou une étude privée pour soi-même, comme l'avait décidé le juge Gibson en première instance. L'absence de jurisprudence sur ce point a forcé les juges à examiner la jurisprudence étrangère, plus spécifiquement celle de l'Angleterre et de la Nouvelle-Zélande. Leur analyse les amène à conclure que:

\section{"Le Barreau n'a d'autre fin quand il copie les ceuvres des éditeurs que de répondre aux fins que poursuivent les demandeurs de copies. Il n'exécute pas cette tâche à son propre avan- tage pécuniaire mais comme un service pour les chercheurs et les étudiants. Son seul but et sa seule intention est d'aider les utilisateurs de la grande bibliothèque dans leur recherche ou étude privée, et il peut dès lors être considéré comme ayant fait sien cet objectif $\rangle^{21}$.}

Toujours sur la notion d'équité comme telle, le tribunal stipule aussi qu'il s'agit toujours d'une question de faits et de degrés, et que chaque cas doit être étudié séparément. Cependant, admettant que le concept d'utilisation équitable n'est pas défini et qu'il est relativement flou, les juges dressent une liste de six considérations empruntées à la jurisprudence canadienne, anglaise et même américaine, dont il faut tenir compte lorsqu'on est appelé à évaluer une utilisation particulière. Ils précisent bien que la liste n'est pas nécessairement exhaustive et qu'aucun élément n'est, en soi, plus déterminant qu'un autre. Ces facteurs sont: le but de l'utilisation, la nature de l'utilisation, la part de l'œuvre utilisée, les autres sources possibles pour utiliser l'œuvre, le type d'œuvre et finalement l'impact de l'utilisation sur l'œuvre.

Quant à la question de la responsabilité des bibliothèques face aux violations possibles du droit d'auteur par l'utilisation des photocopieuses libre-service, la Cour fédérale d'appel est très claire: les juges estiment en effet qu'en mettant des photocopieuses libre-service et une importante collection d'œuvres protégées à la disposition de leur clientèle sans mettre en place des mécanismes de contrôle appropriés pour empêcher les violations du droit d'auteur, le Barreau «de ce fait, sanctionne, appuie ou soutient implicitement les reproductions. En l'espèce, il y a manifestement des éléments de preuve suffisants pour alerter le Barreau, lequel reconnaît, de fait, que les photocopieuses peuvent être utilisées de façon illégale. En conséquence, le Barreau viole le droit des éditeurs d'autoriser les reproductions de leurs ouvres ${ }^{20}$.» 
Enfin, sur la question de la transmission des copies par télécopieur, la Cour fédérale d'appel maintient la décision du juge Gibson en première instance quant à la communication à un destinataire unique plutôt qu' «au public» et rejette la prétention des éditeurs qui ont affirmé dans leur argumentation que le juge Gibson avait commis une erreur en ne décidant pas qu'une série de transmissions à des destinataires uniques pouvait constituer une communication «au public». Tout en admettant que tel pourrait être le cas, les juges considèrent que la preuve devant le juge Gibson et devant eux ne permet pas d'en arriver à une telle conclusion.

\section{LA DÉCISION DE LA COUR SUPRÊME ET SON IMPACT SUR LES BIBLIOTHĖQUES}

Les deux premières instances n'ayant pu s'entendre sur les points majeurs en litige, il devenait évident que le plus haut tribunal du pays allait devoir se prononcer. Il est intéressant de noter ici que, devant l'importance des questions soulevées, l'Association des éditeurs canadiens, la Société québécoise de gestion collective des droits de reproduction (COPIBEC), la Fédération des ordres professionnels de juristes du Canada ainsi que le Canadian Publishers' Council et le Canadian Copyright Licensing Agency (Access Copyright) ont demandé à être entendus comme intervenants devant la Cour suprême.

\section{L'équilibre entre les droits des créateurs et les besoins des utilisateurs}

La décision de la Cour suprême du Canada ne pouvait arriver à un meilleur moment. En effet, le rapport déposé en mai 2004 par le Comité permanent du patrimoine canadien de la Chambre des communes ${ }^{21}$ révèle un préjugé très favorable aux créateurs et une incompréhension malheureuse des besoins des utilisateurs d'œuvres protégées. Pour les membres du Comité, l'octroi de licences et des paiements de redevances correspondants est la solution à tous les problèmes de droit d'auteur et rend le besoin d'exceptions non pertinent. Au moment même où le gouvernement canadien s'apprête à revoir en profondeur sa législation sur le droit d'auteur, compte tenu du nouveau contexte des technologies de l'information, il est rassurant de voir l'insistance que met la Cour suprême sur la nécessité pour la législation sur le droit d'auteur de maintenir un équilibre entre les droits des créateurs et les intérêts des utilisateurs. Pour la deuxième fois en deux ans, le plus haut tribunal du pays explique, comme il l'avait fait dans la cause Théberge:

21. Comité permanent du patrimoine canadien, Rapport intérimaire sur la réforme du droit d'auteur, Ottawa, Chambre des communes, 2004, $34 \mathrm{p}$. «[...]que la Loi sur le droit d'auteur a deux objectifs: La loi est généralement présentée comme établissant un équilibre entre, d'une part, la promotion, dans l'intérêt du public, de la création et de la diffusion des ouvres artistiques et intellectuelles et, d'autre part, l'obtention d'une juste récompense pour le créateur. [...] On atteint le juste équilibre entre les objectifs de politique générale, dont ceux qui précèdent, non seulement en reconnaissant les droits du créateur, mais aussi en accordant l'importance qu'il convient à la nature limitée de ces droits. Lorsqu'ils sont appelés à interpréter la Loi sur le droit d'auteur, les tribunaux doivent s'efforcer de maintenir un juste équilibre entre ces deux objectifs». ${ }^{22}$.

\section{Politique d'accès à l'information documentaire}

Au départ, la Cour suprême retient que le service de photocopie de la Bibliothèque du barreau du Haut-Canada est encadré par une «Politique d'accès à l'information juridique», laquelle comprend des «Lignes directrices», ce qui permet d'évaluer chaque demande à son mérite. Selon les juges :

«La Politique d'accès circonscrit adéquatement le service de photocopie offert. Elle précise que toutes les demandes ne seront pas acceptées. Lorsque la fin poursuivie ne semblera pas être la recherche, la critique, le compte rendu ou l'étude privée, la demande de photocopie sera refusée. En cas de doute quant à la légitimité de la fin poursuivie, il appartiendra aux bibliothécaires de référence de trancher. La Politique d'accès limite l'ampleur de l'extrait pouvant être reproduit, et les bibliothécaires de référence ont le droit de refuser une demande dont la portée excède ce qui est habituellement jugé raisonnable ${ }^{23}$.

À la suite du jugement, on a sauté à des conclusions un peu rapides dans certains milieux, en prétendant que les activités de photocopie dans les bibliothèques étaient pour la plupart des utilisations équitables. Bien que cette affirmation soit juste, on oubliait cependant que la Cour avait quand même tenu compte du fait que les activités de la Bibliothèque du barreau du Haut-Canada étaient encadrées par des politiques d'accès à l'information juridique et des Lignes directrices de service. Il est donc urgent que chaque bibliothèque se dote d'une politique d'accès à l'information documentaire et de lignes directrices de service de photocopie qui expliqueront, tant au personnel qu'à la clientèle, ce qu'on accepte et ce qu'on refuse. Et, comme l'explique le tribunal, la politique devra prévoir

22. Supra note $1, \$ 10$.

23. Ibid., $\$ 73$. 
que des professionnels de l'information documentaire seront en mesure d'évaluer et de décider.

\section{L'originalité d'une ouvre}

Sur la question de ce qui constitue une œuvre «originale» et donc protégée, la Cour suprême est très claire et met fin à toute ambiguité. Pour la Cour, la notion canadienne d'originalité doit se situer au centre des deux courants jurisprudentiels qu'on retrouve actuellement, à savoir, d'un côté, qu'il suffit qu'une œuvre, pour être protégée, émane d'un auteur et soit le résultat d'un certain effort ou d'un labeur, tout en n'étant pas qu'une simple copie d'une autre œuvre et, d'un autre côté, qu'une œuvre doit être créative, c'est-à-dire novatrice ou unique, pour prétendre à une protection légale. Pour la juge McLachlin, qui a rédigé la décision:

«[...] une ceuvre «originale» au sens de la Loi sur le droit d'auteur, est une ouvre qui émane d'un auteur et qui n'est pas une copie d'une autre ouvre. Toutefois, cela ne suffit pas à rendre une ouvre originale. Elle doit en outre être le produit de l'exercice du talent et du jugement d'un auteur. Cet exercice ne doit pas être négligeable au point qu'on puisse le qualifier d'entreprise purement mécanique. Bien qu'une ouvre créative soit par définition «originale» et protégée par le droit d'auteur, la créativité n'est pas essentielle à l'originalité» ${ }^{24 .}$

En appliquant ces principes aux ouvres qu'elle avait devant elle, la Cour suprême a statué que les sommaires, les résumés jurisprudentiels, les lois annotées, les articles de recueils et de revues, et les index analytiques étaient des œuvres protégées. Pour les bibliothèques, cette décision signifie que les abrégés d'articles de revues sont protégés et que leur reproduction multiple dans le cadre des services d'information courante, par exemple, viole le droit d'auteur. Quant aux index, on sait maintenant qu'ils sont protégés, alors que les tables des matières ne répondent pas aux critères d'originalité, puisqu'on n'y retrouve pas l'exercice du talent ou du jugement d'un créateur.

\section{Les photocopieuses libre-service}

La problématique des photocopieuses libre-service est évidemment la question qui intéressait plus particulièrement le monde des bibliothèques, compte tenu des conséquences juridiques possibles d'une poursuite pour violation du droit d'auteur. En effet, depuis plusieurs années déjà, on prétendait que les bibliothèques devaient assumer une responsabilité face aux violations du droit d'auteur qui pouvaient être faites

24. Ibid., $\$ 25$. par les usagers qui utilisent les photocopieuses libreservice qu'on retrouve dans toutes les bibliothèques. Cette prétention reposait principalement sur la cause australienne Moorhouse c. University of New South Wales $^{25}$,où la Haute Cour d'Australie avait justement conclu qu'une bibliothèque universitaire avait enfreint le droit d'auteur en permettant à un de ses usagers d'avoir accès à des photocopieuses libre-service alors qu'elle savait ou avait des raisons de soupçonner que les photocopieuses étaient très probablement utilisées à des fins de contrefaçon. On prétendait aussi que la réforme de la Loi sur le droit d'auteur, en 1999, était venue renforcer cette prétention, alors que le législateur prévoyait maintenant que:

(1) Un établissement d'enseignement, une bibliothèque, un musée ou un service d'archives ne viole pas le droit d'auteur dans le cas où:

- une ouvre imprimée est reproduite au moyen d'une machine à photocopier;

- la machine a été installée dans leurs locaux par eux ou avec leur autorisation à l'usage des enseignants ou élèves ou $d u$ personnel des établissements d'enseignement ou des usagers des bibliothèques, musées ou services d'archives;

- l'avertissement réglementaire a été affiché selon les modalités réglementaires [...].

Application

(2) Le paragraphe (1) ne s'applique que si, selon le cas, en ce qui touche la reprographie:

(a) ils ont conclu une entente avec une société de gestion habilitée par le titulairadu droit d'auteur à octroyer des licences [...] $]^{26}$.

Dans la présente cause, les éditeurs prétendaient justement que le Barreau était responsable des violations du droit d'auteur parce qu'il autorisait tacitement les usagers de sa bibliothèque à copier des œuvres. Or en vertu de l'article 27, paragraphe 1 de la Loi sur le droit d'auteur, autoriser quelqu'un à accomplir un acte que seul le titulaire du droit d'auteur peut accomplir ou autoriser à le faire constitue une violation du droit d'auteur. Pour la Cour suprême, «autoriser» signifie, selon le dictionnaire, "approuver», "sanctionner", «permettre», «favoriser», "encourager». Pour les juges, "ce n'est pas autoriser la violation du droit d'auteur que de permettre la simple utilisation d'un appareil susceptible d'être utilisé à cette fin. Les tribunaux doivent présumer que celui qui autorise une activité ne l'autorise que dans les limites de la légalité 27 ». Quant à l'argument de la cause Moorhouse, que la Cour fédérale d'appel avait retenu, la Cour suprême la rejette parce qu'elle «est incompatible avec

\footnotetext{
25. [1976] Reports of Patent, Design and Trade Mark Cases, 151.

26. Supra note 2, à l'art. 30.2 (1) et (2).

27. Supra note $1, \mathbb{} 38$.
} 
la jurisprudence canadienne et britannique antérieure en la matière ${ }^{28}$ » et il est particulièrement intéressant de noter qu'elle la rejette aussi parce que «l'interprétation retenue dans Moorhouse penche trop en faveur des droits du titulaire et entrave inutilement l'utilisation appropriée des ceuvres protégées pour le bien de l'ensemble de la société29".

Quant aux avis placés près des photocopieuses par la bibliothèque du Barreau avant même que la Loi sur le droit d'auteur l'exige (art. 30.3 (1) (c), les juges considèrent que le Barreau les a justement affichés "pour rappeler aux usagers de la grande bibliothèque que la photocopie de documents de la bibliothèque est assujettie au régime du droit d'auteur ${ }^{30}{ }^{\prime}$. En avertissant les usagers que certaines reproductions peuvent constituer une violation du droit d'auteur, la bibliothèque voulait justement s'assurer que ses équipements seraient utilisés dans le respect de la loi. Pour la Cour suprême, «la Cour d'appel a eu tort de conclure que le Barreau, en affichant l'avis, reconnaissait expressément que les photocopieuses seraient utilisées de façon illicite $^{31}{ }^{2}$. Après avoir indiqué qu'aucun élément de preuve n'avait été présenté pour établir qu'on avait effectivement violé le droit d'auteur par l'utilisation de certains appareils de la bibliothèque, le tribunal va plus loin en indiquant que même si on l'avait fait, "le Barreau n'a pas un contrôle suffisant sur les usagers de la grande bibliothèque pour que l'on puisse conclure qu’il a sanctionné, appuyé ou soutenu la violation $d u$ droit d'auteur. [...] Le Barreau n'exerce pas non plus de contrôle sur les ouvres que les usagers décident de copier, sur les fins auxquelles ils les copient, ni sur les photocopieuses elles-mêmes ${ }^{32}$."

Cette partie de la décision est celle qui a le plus réjoui le monde des bibliothèques. Il faut cependant bien la comprendre de façon à éviter de sauter à de fausses conclusions. Dans un premier temps, cette décision libère toutes les bibliothèques d'un risque de poursuite pour une violation du droit d'auteur qui pourrait être faite par l'utilisation d'un de leur photocopieur. Il y a lieu cependant de demeurer prudent, compte tenu du risque de piège toujours possible qu'on pourrait tendre à certaines bibliothèques. Une violation du droit d'auteur est toujours une question de faits et de degrés, c'est-à-dire qu'un tribunal examinera toujours les circonstances pertinentes. Ainsi, comme c'est le cas dans plusieurs bibliothèques publiques et spécialisées où les photocopieuses sont placées près des comptoirs de prêt, un usager qui utiliserait une photocopieuse pendant une très longue période de temps sans être importuné, alors qu'il devrait apparaitre très clairement au personnel du comptoir

\footnotetext{
28. Ibid., $\$ 41$.

29. Ibid.

30. Supra note $1, \S 44$.

31. Ibid.

32. Supra note $1, \mathbb{} \$ 45$.
}

qu'on photocopie un ouvrage au-delà de toute limite permise, pourrait faire en sorte qu'un tribunal conclue à une violation du droit d'auteur. Il faut se rappeler que la Cour a reconnu que, dans le cas précis de la bibliothèque du barreau, "celle-ci n'avait pas un contrôle suffisant sur les usagers», mais dans un cas où on démontrerait que ce contrôle était possible, la décision pourrait être différente. Dans un deuxième temps, cette décision remet en question les ententes avec les sociétés de gestion collective de droits d'auteurs pour se protéger contre toute poursuite éventuelle pour des violations faites par l'utilisation des photocopieuses libre-service. Il y a lieu de préciser ici que la décision de la Cour suprême n'est pas, en soi, une cause valable de résiliation d'une entente qu'une bibliothèque pourrait déjà avoir avec une société de gestion collective. Cependant, il est clair que la nécessité de négocier de nouvelles ententes en regard des photocopieuses libre-service est remise en question. Les licences demeurent toujours nécessaires pour les bibliothèques qui doivent faire des copies multiples pour les services d'information courante, pour l'usage des étudiants ou pour toute autre raison. Enfin, l'obligation légale d'afficher un avertissement réglementaire tel que prévu à l'article 30.3 (1) (c) demeure. De plus, le texte de cet avis n'est pas laissé au choix de la bibliothèque, mais doit être celui prévu au règlement ${ }^{33}$.

\section{L'utilisation équitable d'une ouvre protégée}

La question de l'utilisation équitable fait l'objet d'une analyse en profondeur par la Cour suprême et on doit admettre que les juges, de façon assez surprenante, ont été assez loin. Encore ici, le plus haut tribunal du pays, dans une nouvelle approche jurisprudentielle, réitère le principe que l'équilibre entre les droits des créateurs et les intérêts des utilisateurs doit prévaloir. Il faut bien comprendre que l'utilisation équitable en matière de droit d'auteur a toujours été considérée comme un moyen de défense plutôt qu'une exception légale. En effet, dans les causes où la notion d'utilisation équitable était en jeu, le défendeur devait démontrer que son utilisation pouvait être considérée comme équitable. Dans la présente cause, on remet ce principe en question:

«[...] il est peut-être plus juste de considérer cette exception [l'utilisation équitable] comme une partie intégrante de la Loi sur le droit d'auteur plutôt que comme un simple moyen de défense. [...] À l'instar des autres exceptions que prévoit la Loi sur le droit d'auteur, cette exception correspond à un droit des utilisateurs. Pour maintenir un juste équilibre entre les droits des titulaires du droit d'auteur

33. Règlement sur les cas d'exception à l'égard des établissements d'enseignement, des bibliothèques, des musées et des services d'archives, D.O.R.S./99-325, art. 8. 
et les intérêts des utilisateurs, il ne faut pas l'interpréter restrictivement. ${ }^{34}{ }^{\text {» }}$

La Cour poursuit sa réflexion en affirmant que l'exception créée par l'article 29, c'est-à-dire l'utilisation équitable, peut toujours être invoquée et spécifie qu' «ainsi, une bibliothèque peut toujours tenter d'établir que son utilisation d'une ouvre protégée est équitable, suivant l'art. 29 de la Loi sur le droit d'auteur. C'est seulement dans le cas où elle n'est pas en mesure de prouver l'application de cette exception qu'il lui faut s'en remettre à celle que prévoit l'art. 30.2 au bénéfice des bibliothèques ${ }^{35}$." La Cour suprême reprend par la suite et soutient l'argumentation de la Cour fédérale d'appel à l'effet "que la recherche ne se limite pas à celle effectuée dans un contexte non commercial ou privéz $^{36}$ ». Il s'agit là d'un aspect très important pour les bibliothèques spécialisées faisant partie d'organisations commerciales ou industrielles. Celles-ci s'étaient vu refuser la reconnaissance du statut de bibliothèque au sens de la Loi sur le droit d'auteur, lors de la réforme de 1999, ce qui les empêchait d'être éligibles aux nouvelles exceptions prévues pour les bibliothèques. De plus, plusieurs soutenaient que les usagers de ces bibliothèques ne pouvaient prétendre à la défense de l'utilisation équitable en raison du caractère lucratif de leurs activités de recherche. La Cour suprême règle également le questionnement que les bibliothèques à caractère non lucratif pouvaient avoir lorsqu'elles répondaient à des demandes de services de livraison de documents pour cette catégorie de bibliothèques.

\section{Les critères à considérer pour juger de l'utilisation équitable}

La Cour suprême fait également siens les critères que la Cour fédérale d'appel avait identifiés pour déterminer ce qui pouvait rendre une utilisation équitable. Il est important de s'y arrêter parce que le personnel des bibliothèques pourrait être appelé à les utiliser pour évaluer certaines situations. On doit se rappeler que la Cour a clairement établi que les «bibliothécaires de référence» ont une responsabilité professionnelle dans l'application de la notion d'utilisation équitable. Sur le critère concernant le but de l'utilisation, la Cour précise que «l'utilisation ne sera manifestement pas équitable si la fin poursuivie n'est pas celles que prévoit la Loi sur le droit d'auteur, savoir la recherche, létude privée, la critique, le compte rendu ou la communication de nouvelles. [...] Il ne faut pas interpréter ces fins restrictivement, sinon les droits des utilisateurs pourraient être indûment restreints ${ }^{37}$." Dans ses considérations pour juger de la preuve qu'on lui présente, la Cour reconnaît que la Politique d'accès

\footnotetext{
34. Supra note $1, \$ 48$.

35. Ibid., $\$ 49$.

36. Ibid., $\$ 51$.

37. Ibid., $\$ 54$.
}

et les Lignes directrices du service de photocopie de la Bibliothèque du barreau précisent exactement les fins pour lesquelles on acceptera de faire des photocopies. Quant au critère qui concerne la nature de l'utilisation, la Cour suggère quelques pistes comme "[1]orsque de multiples copies sont diffusées largement, l'utilisation tend à être inéquitable. Toutefois, lorsqu'une seule copie est utilisée à une fin légitime en particulier, on peut conclure plus aisément que l'utilisation était équitable. Si la copie de l'ouvre est détruite après avoir été utilisée comme prévu, cela porte également à croire qu'il s'agissait d'une utilisation équitable ${ }^{38}$.» Toujours en examinant spécifiquement le cas de la bibliothèque du Barreau, la Cour constate que la politique précise clairement qu'on fournit une seule copie. Un autre critère à considérer est l'ampleur de l'utilisation, et dans ce cas, les juges précisent d'abord que lorsqu'une infime partie de l'œurre est utilisée, il n'est pas nécessaire d'entreprendre une analyse relative du caractère équitable de l'utilisation. Ils poursuivent leur analyse en mentionnant que "[1]'ampleur de l'extrait peut aussi être plus ou moins équitable selon la fin poursuivie. Par exemple, aux fins de recherche ou d'étude privée, il peut être essentiel de reproduire en entier un exposé universitaire ou une décision de justice. Cependant, lorsqu'une ouvre littéraire est reproduite aux fins de critique, il ne sera vraisemblablement pas équitable de la copier intégralement ${ }^{39}$.» On peut facilement conclure à partir de cette considération que la reproduction d'articles de revues savantes à des fins de recherche ou d'étude privée est une utilisation équitable. Encore une fois, la Cour se dit satisfaite de constater que la Politique d'accès et les Lignes directrices prévoient que toute demande excédant $5 \%$ est référée à un bibliothécaire de référence qui juge de l'équité de la demande. Le critère des solutions de rechange à l'utilisation est par la suite examiné par le tribunal. La Cour considère d'abord le fait qu'on ne peut quand même pas raisonnablement s'attendre à ce que tous les usagers se rendent à la bibliothèque pour y faire leur recherche et leurs copies, d'où la nécessité du service de photocopie et de livraison de documents. Il est important de noter ici que la Cour suprême considère, eu égard à ce critère, que:

"La possibilité d'obtenir une licence n'est pas pertinente pour décider du caractère équitable d'une utilisation. [...] Si, comme preuve du caractère inéquitable de l'utilisation, le titulaire du droit d'auteur ayant la faculté d'octroyer une licence pour l'utilisation de son ceuvre pouvait invoquer la décision d'une personne de ne pas obtenir une telle licence, il en résulterait un accroissement de son monopole sur l'ouvre qui serait incompatible avec l'équilibre queétablit la Loi sur le droit d'auteur

38. Ibid., $\$ 55$.

39. Ibid., $\$ 56$ 
entre les droits du titulaire et les intérêts de

l'utilisateur.» ${ }^{40}$.

Il s'agit là d'une affirmation d'une très grande importance pour les bibliothèques qui ont la plupart du temps signé des ententes avec les sociétés de gestion collective de droits d'auteur, par crainte de poursuites plutôt que par conviction que certaines de leurs activités étaient inéquitables en regard du droit d'auteur. Pour la Cour suprême du Canada, le fait, pour une bibliothèque, de ne pas acquérir une licence ne permet pas pour autant aux sociétés de gestion collective de prétendre que l'utilisation des œuvres protégées que fait cette bibliothèque est inéquitable. Les juges passent rapidement sur la nature de l'ouvre, ne considérant pas ce critère comme nécessairement décisif. Comme exemple, on mentionne que la reproduction d'une œuvre confidentielle pourrait être inéquitable. Finalement le sixième critère analysé par les juges concerne l'effet de l'utilisation de l'oeuvre. Ainsi, «[1] a concurrence que la reproduction est susceptible d'exercer sur le marché de l'ouvre originale peut laisser croire que l'utilisation n'est pas équitable ${ }^{41}$ ».

Enfin, toujours sur la question de l'utilisation équitable, la Cour suprême ouvre une nouvelle voie jurisprudentielle très rassurante pour les bibliothèques en affirmant qu'une bibliothèque n'a pas à démontrer que chacune des utilisations individuelles des œuvres protégées qu'elle fait est équitable. En effet, pour le plus haut tribunal du pays, une bibliothèque peut invoquer l'exception de l'utilisation équitable en démontrant que ses politiques et ses pratiques sont axées sur la recherche et l'étude privée. La juge McLachlin, en parlant de l'article 29 de la Loi sur le droit d'auteur, qui traite de l'utilisation équitable, dit ceci: "Les termes employés sont généraux. "Utilisation" ne renvoie pas à un acte individuel, mais bien à une pratique ou un système. Cela est compatible avec l'objet de l'exception au titre de l'utilisation équitable, qui est de faire en sorte que la faculté des utilisateurs d'utiliser et de diffuser des œuvres protégées ne soit pas indûment limitée $e^{42}$."

Autant pour chacun des critères à considérer que pour l'utilisation équitable considérée comme système ou pratique, la Cour s'appuie sur la Politique d'accès et les Lignes directrices de la Bibliothèque du barreau du Haut-Canada pour s'assurer que le service de photocopie offert est équitable. Il faut donc rappeler l'importance, pour les bibliothèques, de mettre en place des politiques écrites et publicisées ainsi que des lignes directrices encadrant leur service de livraison de documents. La Cour suprême du Canada en fait une exigence pour celles qui veulent invoquer l'exception de l'utilisation équitable.

\section{La transmission de copies par télécopieur}

Sur cette question, les trois niveaux de cour s'entendent, la Cour suprême venant confirmer que la transmission des copies par télécopieur à un seul destinataire ne constituait pas une «communication au public» et ne violait pas le droit d'auteur. Il faut bien prendre note cependant que la Cour suprême précise que «la transmission répétée d'une copie d'une même ouvre à de nombreux destinataires pourrait constituer une communication au public et violer le droit d'auteur ${ }^{43}$ ». Les bibliothèques qui offrent des services d'information courante à leurs usagers, et qui transmettent leur bulletin par télécopieur ou par tout autre moyen, devront tenir compte de cette décision de la Cour suprême. En effet, eu égard à la Loi sur le droit d'auteur, le terme public ne signifie pas «grand public», mais bien tout ce qui est à l'extérieur du contexte familial. Dans ce sens, le personnel d'une organisation constitue un public.

\section{CONCLUSION}

Cette décision du plus haut tribunal du pays doit réjouir le monde des bibliothèques, tant le personnel que les usagers. À plusieurs égards, on doit parler de victoire et de pas importants dans la reconnaissance des besoins légitimes des utilisateurs d'œuvres protégées. Considérant que le débat se situe actuellement presque exclusivement au niveau des considérations de nature économique et que l'émission de licences apparaît à certains comme la solution à tous les problèmes de droit d'auteur, il est rassurant de voir la Cour suprême du Canada venir rappeler certains grands principes de base du droit d'auteur. Enfin, au moment où le parlement canadien s'apprête à débattre une nouvelle loi sur le droit d'auteur qui tiendra compte de l'environnement numérique, où les enjeux économiques sont énormes, la décision de la Cour suprême vient rappeler au législateur que toute législation sur le droit d'auteur doit rechercher un juste équilibre entre les droits des créateurs et l'intérêt public, qui se manifeste ici par le droit à un accès facile et non restrictif. La décision de la Cour suprême ne règle pas tout. Certaines questions restent en suspens et de nouvelles ont été soulevées. Mais il s'agit sans contredit d'une décision majeure aux conséquences importantes pour les bibliothèques. $\odot$ 